08

\title{
Изготовление и исследование свойств вогнутого кристаллического зеркала для проекта КОРТЕС
}

\author{
(C) А.А. Ахсахалян, ${ }^{1}$ А.Д. Ахсахалян, ${ }^{1}$ С.А. Гарахин, ${ }^{1}$ Н.Ф. Ерхова, ${ }^{2}$ А.С. Кириченко, ${ }^{2}$ С.В. Кузин, ${ }^{2}$ \\ Н.Н. Салащенко, ${ }^{1}$ М.Н. Торопов, ${ }^{1}$ Н.И. Чхало ${ }^{1}$ \\ ${ }^{1}$ Институт фризики микроструктур РАН, \\ 607680 Нижний Новгород, Россия \\ ${ }^{2}$ Физический институт им. П.Н. Лебедева РАН, \\ 199991 Москва, Россия \\ e-mail: akh@ipm.sci-nnov.ru
}

Поступило в Редакцию 28 марта 2019 г.

В окончательной редакции 28 марта 2019 г.

Принято к публикации 15 апреля 2019 г.

\begin{abstract}
Описана методика изготовления и представлены результаты исследования рентгенооптических свойств вогнутого сферического зеркала из кристаллического кварца. Радиус кривизны составил $1630 \mathrm{~mm}$. Форма поверхности зеркала была изучена на интерферометре с дифракционной волной сравнения. Показано, что во всей области обработки максимальное отклонение от ближайшей сферы составило около $0.2 \mu$ m, а корень квадратный из среднеквадратического значения - $34 \mathrm{~nm}$, что обеспечивает высокое качество изображений с угловой ошибкой на уровне $2^{\prime \prime}$ (angular seconds). Определены коэффициенты отражения (около 7\%) и нижняя оценка спектральной селективности $(\lambda / \delta \lambda \approx 1775)$ в окрестности длины волны $\lambda=0.834 \mathrm{~nm}$, близкой к рабочим длинам волн дублета $\operatorname{MgXII}(\lambda=0.8418$ и $0.8423 \mathrm{~nm})$. При изгибе кристаллического зеркала в пределах точности измерений изменение положения брэгговского пика и спектральной селективности не обнаружено.
\end{abstract}

Ключевые слова: рентгеновское зеркало, сферическое кристаллическое рентгеновское зеркало, рентгеновский спектрограф.

DOI: $10.21883 / J T F .2019 .11 .48342 .124-19$

\section{Введение}

Космический эксперимент КОРТЕС [1] предназначен для исследования солнечной короны на фазе максимума и спада цикла солнечной активности, которая характеризуется большим количеством вспышек. Исходя из научных задач эксперимента, исследования должны проводиться в первую очередь в спектральных диапазонах рентгеновской и экстремальной ультрафиолетовой части спектра, где доминируют линии многозарядных ионов, характерные для „горячей“ вспышечной плазмы с температурой в несколько миллионов градусов. Особый интерес представляет регистрация солнечных изображений в монохроматических горячих линиях, так как это сильно упрощает температурную диагностику наблюдаемой плазмы и позволяет выделить именно вспышечные процессы на Солнце. Одним из таких диапазонов является спектральная область вблизи $0.842 \mathrm{~nm}$, где расположен дублет иона MgXII. Эти линии возбуждаются при температуре выше 4 миллионов градусов (МК), и максимум излучения соответствует $10 \mathrm{MK}$. Для регистрации этого дублета предлагается использовать спектрогелиометр на основе кристаллического зеркала [2]. В качестве материала зеркала выбран кристаллический кварц, с отражающей кристаллографической плоскостью $10 \overline{1} 0$ $d=0.42548 \mathrm{~nm}$ [3]. Особенностью этого прибора яв- ляется узкий диапазон регистрации $0.842 \pm 0.01 \mathrm{~nm}$, в котором находятся только линии иона MgXII.

Схема прибора представлена на рис. 1. Основным элементом прибора является кристаллическое зеркало, которое представляет собой сферически изогнутую пластину кристаллического кварца. В фокусе зеркала расположен детектор на основе ПЗС-матрицы обратного падения с прямой регистрацией рентгеновского излучения. Радиус кривизны зеркала составляет $1670 \mathrm{~mm}$, апертура рабочей части кристалла $60 \times 40 \mathrm{~mm}$. Угол падения, отсчитываемый от поверхности, равен $82.1^{\circ}$. Для блокирования видимого излучения в приборе установлено два тонкопленочных фильтра - на входной апертуре и вблизи детектора. Прибор в целом планируется изготовить силами ФИАН. Зеркало планируется изготовить в ИФМ РАН.

\section{Изготовление зеркала и исследование его свойств}

Для изготовления зеркала мы использовали метод оптического контакта (ОК) [4]. Этот метод широко применяется для изготовления фокусирующих рентгеновских зеркал цилиндрической и сферической формы [5-8]. $\mathrm{B}$ настоящей работе в качестве подложки использовался кварц с вогнутой полированной сферической поверхно- 


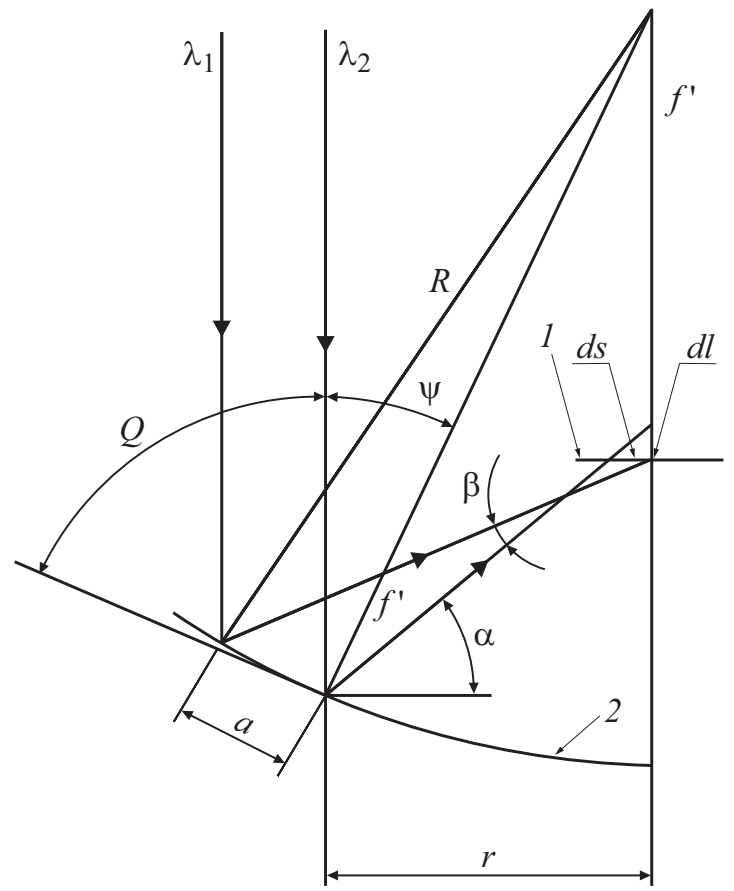

Рис. 1. Принципиальная оптическая схема телескопаспектрогелиометра: $a-$ радиальное расстояние между кольцевыми зонами дифракции для $\lambda_{1}$ и $\lambda_{2}, r-$ радиус кольцевой зоны дифракциидля $\lambda_{2}, f^{\prime}-$ фокусное расстояние кристалла, $\varphi-$ угол скользящего падения, $R-$ радиус кривизны кристаллического зеркала, $d s-$ поперечная сферическая аберрация, $d l$ - продольная сферическая аберрация, 1 - плоскость детектора, 2 - сферический кристалл.

стью с радиусом кривизны $R=1670 \mathrm{~mm}$. На подложку методом ОК приклеивается тонкая $(350 \mu \mathrm{m})$ пластина из кристаллического кварца, полированная с обеих сторон.

Фотография готового зеркала приведена на рис. $2, a$. Визуально, по цвету интерференционной картины, формируемой в результате интерференции двух волн, отраженных от поверхностей кварцевой подложки и тыльной стороны изогнутого кристалла, зазор между ними не превышает $20 \mathrm{~nm}$, что говорит о высоком качестве ОК. Для измерения отклонения формы изготовленной поверхности от сферы использовался разработанный в ИФМ РАН интерферометр с дифракционной волной сравнения (ИДВС) [9]. В приборе в качестве источника эталонной сферической волны используется торец одномодового оптического волокна. Оптическая схема интерферометра приведена на рис. 3. Излучение $\mathrm{He}-\mathrm{Ne}$-лазера $1(\lambda=632.8 \mathrm{~nm})$ заводится в оптоволокно, поступает в блок 2 с делителем света, фазосдвигающим элементом и поляризационными контроллерами. На выходе блока 2 формируется два канала. К одному каналу подключается первый источник эталонной сферической волны 3.1, ось которого совпадает с оптической осью исследуемого образца 7 , к другому каналу второй источник эталонной сферической волны 3.2 , ось которого совпадает с оптической осью наблюдательной системы 4. Сферический фронт от источника 3.2 используется в качестве эталонного, а сферический фронт от источника 3.1 в качестве рабочего, фронт от которого при отражении от исследуемого образца 7 плоским зеркалом 6 направляется в сторону наблюдательной системы 4. Полученная интерференционная картина регистрируется CCD-камерой 5 и обрабатывается на компьютере.

На рис. 2 представлена полученная интерферограмма и наложенная на нее карта отклонения от сферы поверх-

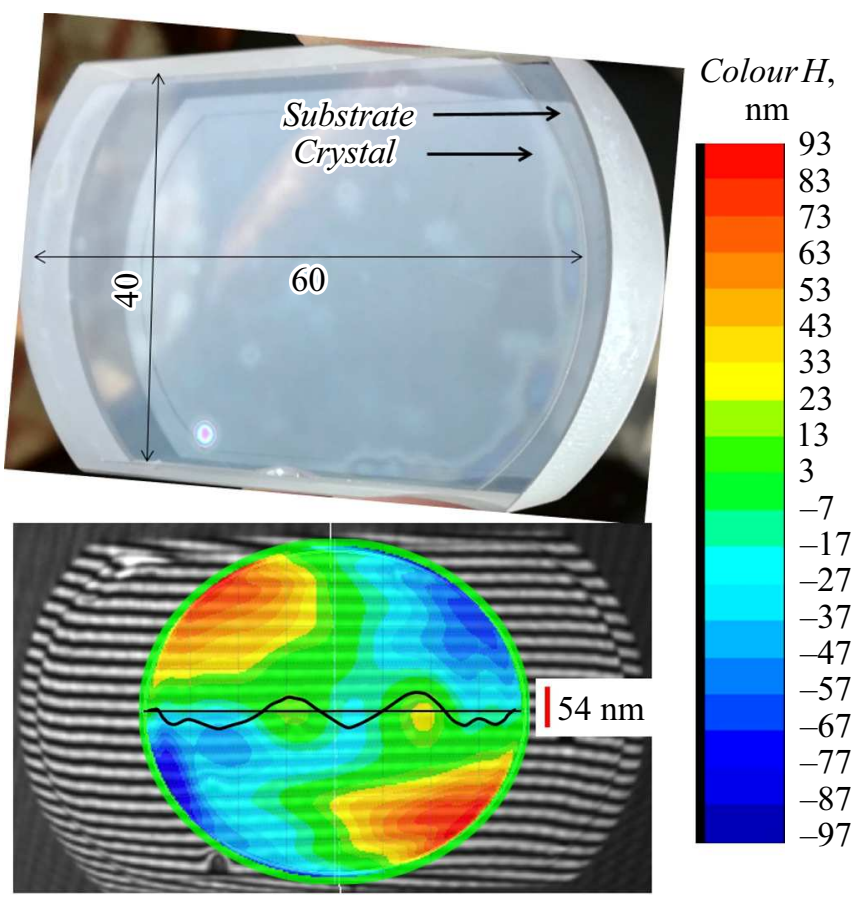

Рис. 2. $a-$ фотография зеркала. $b-$ интерферограмма поверхности, карта отклонения поверхности от сферы и отклонение профиля от окружности в центральном сечении.

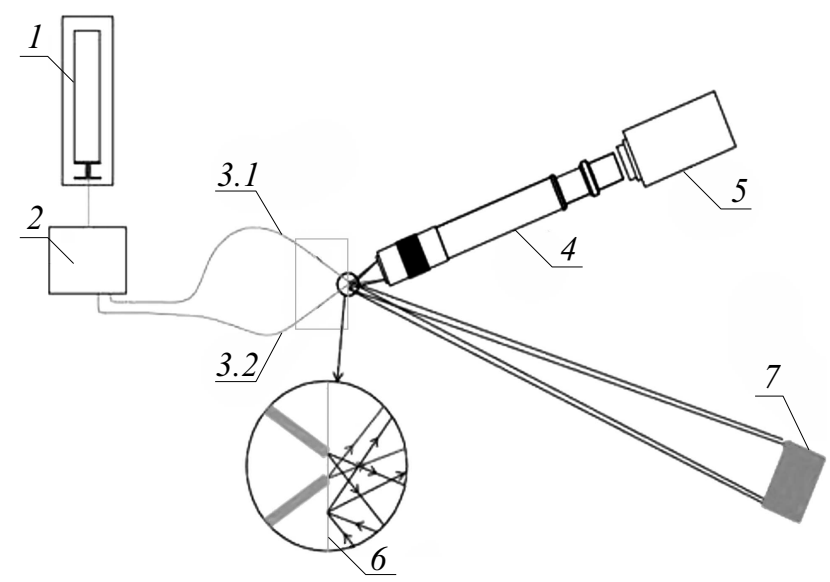

Рис. 3. Схема измерений с помощью ИДВС: 1 $\mathrm{He}-\mathrm{Ne}$-лазер; 2 - блок формирования 2-х каналов с поляризационными контроллерами; 3.1 и $3.2-$ источник сферической волны; 4 - наблюдательная система; 5 - CCD-камера, $6-$ плоское зеркало; 7 - исследуемый образец. 


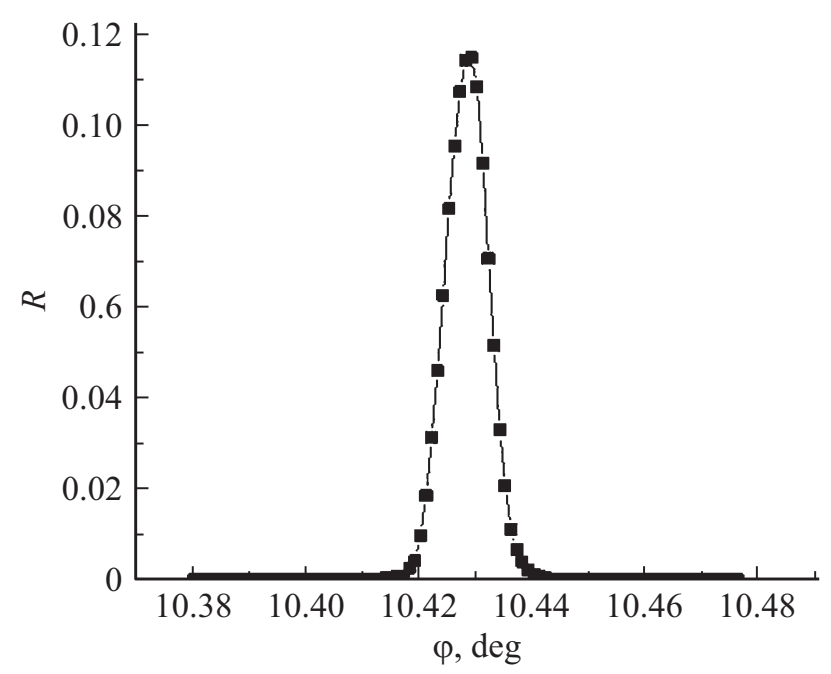

Рис. 4. Угловая зависимость коэффициента отражения кристаллического кварца, плоскость $10 \overline{1} 0 d=0.42548 \mathrm{~nm}$, измеренная на длине волны $0.154 \mathrm{~nm}$.

ности зеркала в центральной части диаметром $40 \mathrm{~mm}$. Поверхность рассчитывалась аппроксимацией экспериментальных точек полиномами Цернике девятого порядка по методике [10]. Максимальное и среднеквадратичное отклонение составило 0.2 и $0.034 \mu \mathrm{m}$ соответственно. Отклонение профиля от сферы в центральном (рабочем) сечении составило $54 \mathrm{~nm}$, а отклонение локального угла от сферы не превышает $1 \cdot 10^{-5} \mathrm{rad}\left(\sim 2^{\prime \prime}\right.$, angular seconds).

Измерения в жестком рентгеновском диапазоне проводились на четырехзеркальном дифрактометре высокого разрешения PANalytical E'Xpert PRO, в мягком - на лабораторном рефлектометре, использующем разборную рентгеновскую трубку в качестве источника рентгеновского излучения и решеточный спектрометр-монохроматор РСМ 500 [11]. Экспериментально измерялись угловые зависимости коэффициента отражения на характеристических линиях $\mathrm{Cu} K \alpha$ с длиной волны $\lambda=0.154 \mathrm{~nm}$ и $\mathrm{Al} K \alpha, \lambda=0.834 \mathrm{~nm}$.

Измерения на длине волны $0.154 \mathrm{~nm}$ проводились с целью оценки качества полировки кристалла и точного определения угла между рабочей крисллографической плоскостью и поверхностью зеркала. Последнее крайне важно для оптической юстировки зеркала в телескопе.

Отражательные характеристики кристалла в мягком рентгеновском диапазоне измерялись как до установки кристалла на сферическую подложку, так и на подложке (изогнутый). Это исследование преследовало две цели. Первая, оценка возможной деформации кристаллической решетки в результате изгиба и, как следствие, изменения величины брэгговского угла. Вторая, определение абсолютного значения коэффициента отражения на длине волны, максимально приближенной к рабочей, для определения энергетической эффективности телескопа.
На рис. 4 приведена измеренная на длине волны $0.154 \mathrm{~nm}$ угловая зависимость от коэффициента отражения от кристаллического полированного кварца в окрестности брэгговского пика, плоскость $10 \overline{1} 0$, $d=0.42548 \mathrm{~nm}$. Достаточно высокий коэффициент отражения, отсутствие диффузного рассеяния указывают на высокую степень полировки. Угол между кристаллографической плоскостью и поверхностью составил $1^{\prime}$ (angular minute).

На рис. 5 приведены угловые зависимости коэффициента отражения, измеренные на характеристической линии $\mathrm{Al} K \alpha(\lambda=0.834 \mathrm{~nm})$. Квадратиками обозначена кривая, соответствующая отражению от плоской пластины, кругами - от сферы. Из рисунка видно, что в пределах точности измерений ни коэффициент отражения, ни полуширина кривой отражения, ни положение брэгговского пика не изменились после приклейки на ОК кристалла на сферическую подложку. Максимум коэффициента отражения при скользящем угле падения $\theta=78.590^{\circ}$ составил около $7 \%$, ширина пика на полувысоте $\Delta \theta=0.16^{\circ}$.

Формально спектральная селективность кристалла, вычисленная из соотношения

$$
\lambda / \delta \lambda=\tan \varphi_{B r} / \Delta \varphi_{B r}
$$

составила $\lambda / \delta \lambda \approx 1775$, что вполне достаточно для решения задачи солнечной спектроскопии.

В действительности спектральное разрешение кристалла даже выше. На кривых отражения можно обратить внимание на асимметрию кривых отражения. Наблюдается некоторое уширение кривой в области больших углов. Причина этого уширения является то, что в эксперименте частично разрешается дублет

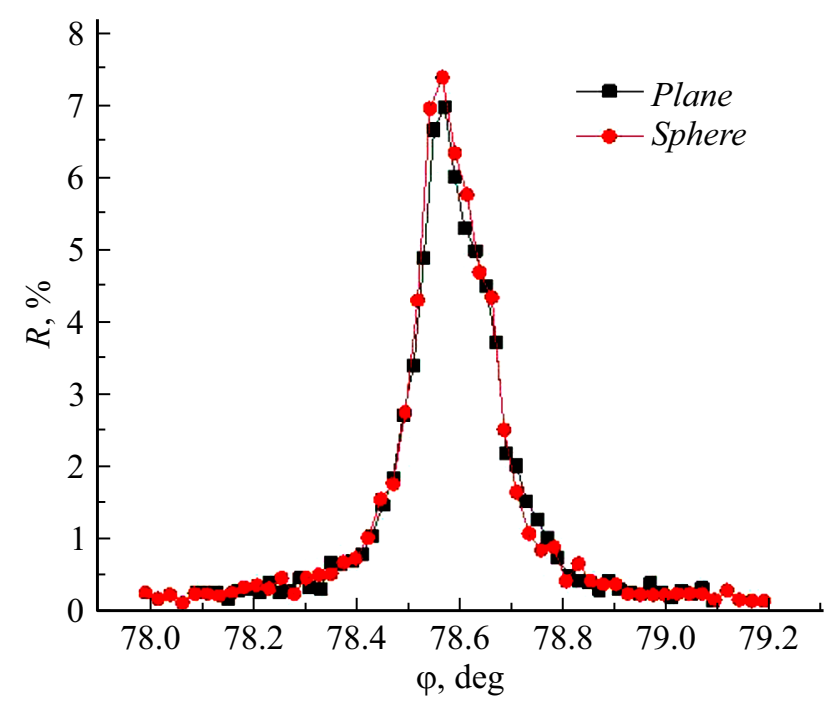

Рис. 5. Угловая зависимость коэффициента отражения кристаллического кварца, плоскость $10 \overline{1} 0 d=0.42548 \mathrm{~nm}$, измеренная на характеристической линии $\mathrm{Al} K \alpha(\lambda=0.834 \mathrm{~nm})$. Квадратиками обозначена кривая, соответствующая отражению от плоской пластины, кругами — от сферы. 
$\mathrm{Al} K \alpha_{1},(\lambda=0.833934 \mathrm{~nm}$ - основной пик $)$ и $\mathrm{Al} K \alpha_{2}$, $(\lambda=0.834173 \mathrm{~nm}$ - уширение в области больших углов).

\section{Заключение}

Работа посвящена изготовлению и изучению рентгенооптических характеристик кристаллического кварцевого зеркала со сферической формой поверхности, и предназначенного для изображающей спектроскопии Солнца в рентгеновских линиях иона магния, дублет $\operatorname{MgXII~}(\lambda=0.8418$ и $0.8423 \mathrm{~nm})$. Основными результатами работа стало следующее.

Во-первых, показано, что разработанный метод ОК позволяет изготавливать кристаллические кварцевые сферические зеркала с радиусом кривизны, по крайне мере $R=1670 \mathrm{~mm}$. Качество отражающей поверхности оказалось довольно высоким. По данным интерферометрии максимальное отклонение поверхности от ближайшей сферы в центральном сечение составило $54 \mathrm{~nm}$, что соответствует максимальной угловой ошибке около $2^{\prime \prime}$ (angular seconds). По параметру среднеквадратического отклонения от ближайшей сферы ошибка составляет $34 \mathrm{~nm}$. Таким образом, изготовленное зеркало обеспечивает высокое качество изображения.

Относительная легкость организации ОК на этой сфере позволяет надеяться на то, что данный метод может применяться и для подложек с меньшими радиусами кривизны.

Во-вторых, посадка кристалла на сферическую подложку не привела к заметному изменению ни коэффициента отражения, ни положения, ни ширины брэгговского пика, что позволяет надежно предсказывать рентгенооптические характеристики такого зеркала.

В-третьих, видно, нижняя оценка спектральной селективности изготовленного кристаллического зеркала составляет $\lambda / \delta \lambda \approx 1775$. В действительности она даже выше и позволит надежно разрешить даже дублет линий ионов $\mathrm{MgXII}$, длины волн $\lambda=0.8418$ и $0.8423 \mathrm{~nm}$. Этот вывод следует из эксперимента, так как частично разрешенный дублет $\mathrm{Al} K \alpha_{1}$ и $\mathrm{Al} K \alpha_{2}$ имеет разность длин волн $0.0002 \mathrm{~nm}$, в то время как дублет ионов магния $0.0005 \mathrm{~nm}$.

Таким образом, разработанное зеркало может с успехом применяться в составе научной аппаратуры КОРТЕС для изучения короны Солнца с высоким спектральным и пространственным разрешением, и эффективностью регистрации ретгеновского излучения.

\section{Финансирование работы}

Работа выполнена при финансовой поддержке Министерства образования и науки РФ в рамках Соглашения № 075-02-2018-182 (RFMEFI60418X0202).

\section{Благодарность}

Работа выполнена с использованием оборудования ЦКП ИФМ РАН

\section{Конфликт интересов}

Авторы заявляют, что у них нет конфликта интересов.

\section{Список литературы}

[1] Shestov S.V., Ulyanov A.S., Vishnyakov E.A., Kuzin S.V., Pertsov A.A. // Proc. SPIE. 2014. Vol. 9144. P. 91443G. DOI: $10.1117 / 12.2055946$

[2] Kuzin S.V., Andreev E.E., Korneevet V.V., Krutov V.V., Mitropoisky M.M., Pertzov A.A., Stasevich V.N., Sobelman I.I., Tindo I.P., Zhitiük I.A. // $X$-Ray and Ultraviolet Spectroscopy and Polarimetry. 1994. Vol. 2283. P. 242. DOI: $10.1117 / 12.193192$

[3] Блохин М.А., Швейщер И.Г. Рентгеноспектральный справочник. М.: Наука, 1982. 374 с.

[4] Обреимов И.В. // ЖЭТФ. 1957. Т. 32. Вып. 2. С. 185.

[5] Schuster M., Gobel H. // J. Phys. D: Appl. Phys. 1995. Vol. 28. P. A270. DOI: 10.1088/0022-3727/28/4A/053

[6] Akhsakhalyan A.D., Chkhalo N.I., Kharitonov A.I. // NIM A. 2001. Vol. 470. P. 142. DOI: 10.1016/S0168-9002(01)01027-0

[7] Ахсахалян А.А., Ахсахалян А.Д., Волгунов Д.Г., Гапонов С.В., Короткова Н.А., Мазо Л.А., Миронов В.Л., Муравьев В.А., Салащенко Н.Н., Харитонов А.И. // Поверхность. Рентгеновские, синхротронные и нейтронные исследования. 2003. № 1. С. 78-80.

[8] Akhsakhalyan A.A., Akhsakhalyan A.D., Kharitonov A.I., Kluenkov E.B., Muravev V.A., Salashchenko N.N. // CEJP. 2005. Vol. 3. N 2. P. 163-177. DOI: $10.2478 / \mathrm{BF} 02475584$

[9] Svechnikov M.V., Chkhalo N.I., Toropov M.N., Salashchenko N.N., Zorina M.V. // Opt. Lett. 2015. Vol. 40. N 2. C. 159. DOI: $10.1364 /$ OL.40.000159

[10] Svechnikov M.V., Chkhalo N.I., Toropov M.N., Salashchenko N.N. // Opt. Express. 2015. Vol. 23. N 11. P. 14677-14694. DOI: 10.1364/OE.23.014677

[11] Bibishkin M.S., Chekhonadskih D.P., Chkhalo N.I., Klyuenkov E.B., Pestov A.E., Salashchenko N.N., Shmaenok L.A., Zabrodin I.G., Zuev S.Yu. // Proceed. SPIE. 2004. Vol. 5401. P. 8-15. DOI: 10.1117/12.556949 